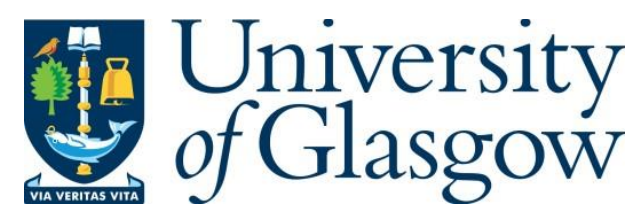

Li, W., Li, X., Ren, J. and Jiang, H. (2018) Experimental investigation of wall thickness and hole shape variation effects on full-coverage film cooling performance for a gas turbine vane. Applied Thermal Engineering, 144, pp. 349-361.

There may be differences between this version and the published version. You are advised to consult the publisher's version if you wish to cite from it.

http://eprints.gla.ac.uk/175284/

Deposited on: 8 January 2019

Enlighten - Research publications by members of the University of Glasgow http://eprints.gla.ac.uk 


\title{
EXPERIMENTAL INVESTIGATION OF WALL THICKNESS AND HOLE SHAPE VARIATION EFFECTS ON FULL-COVERAGE FILM COOLING PERFORMANCE FOR A GAS TURBINE VANE
}

\author{
Weihong Li, Jing Ren, Hongde Jiang \\ Institute of Gas Turbine, Department of Energy and Power Engineering, \\ Tsinghua University, Beijing, China. \\ Liwh13@mails.tsinghua.edu.cn
}

\begin{abstract}
The effects of wall thickness and hole shape variation on a full-coverage film cooled turbine vane are investigated in a stationary and linear cascade utilizing the pressure sensitive paint technique. The varied wall thickness produces hole lengthto-diameter ratio (L/D) in a range from $\mathrm{L} / \mathrm{D}=2$ to 5 , and holes tested include simple angle hole, compound angle hole, and fan-shaped hole. Five rows of holes are provided on the pressure side while three rows of holes are provided on the suction side, with six rows of cylindrical holes drilled on the leading edge to construct showerhead film cooling. The tested blowing ratios for the showerhead, pressure side, and suction side range from 0.25 to 1.5 , with a density ratio of 1.5 . The freestream Reynolds number is $1.35 \times 10^{5}$, based on the axial chord length and the inlet velocity, with a freestream turbulence intensity level of $3.5 \%$ at the cascade inlet. The results indicate that the wall thickness variation produces significant influence on the pressure side film cooling effectiveness, while only marginal effect on the showerhead and suction side film cooling. Also observed is that the fan-shaped hole generates the highest film cooling effectiveness on pressure or suction side. Also discussed is the surface curvature effect, combining with effects of wall thickness and hole shape variations, on the film cooling effectiveness in comparison to the flat-plate data.
\end{abstract}

\section{Introduction}

Modern heavy-duty gas turbines or aero-engines are requiring higher pressure ratio and turbine inlet temperature to achieve more power generation and higher thermal efficiencies. Typical turbine inlet temperature has increased up to $2100 \mathrm{~K}$ for aero-engines which generates higher demand of turbine cooling. Common cooling techniques include internal cooling and film cooling. Coolant passes internal channels with different turbulent features and then injects through discrete holes to form a protective film on the turbine blade surface. Recently, some new blade concepts are proposed, for example, near wall cooling or double wall airfoil cooling [1], where small cooling cavities require impingement with low distance [2-4] and thin-wall outer foil requires discrete film holes with short length-todiameter ratio [5-7]. Figure 1 presents a schematic drawing of the double wall airfoil, where the outer foil is covered with film cooling holes.

Designing an efficient turbine film cooling configuration is becoming more complicated by several factors, including geometrical parameters, e.g., hole shape, length-to-diameter ratio, hole spacing, and flow parameters, e.g., blowing ratio, momentum ratio, Mach number, Reynolds number, turbulence intensity and scale to name but a few. These geometrical and flow parameter effects on the turbine film cooling have been studied in the literature through experimental and numerical methods.

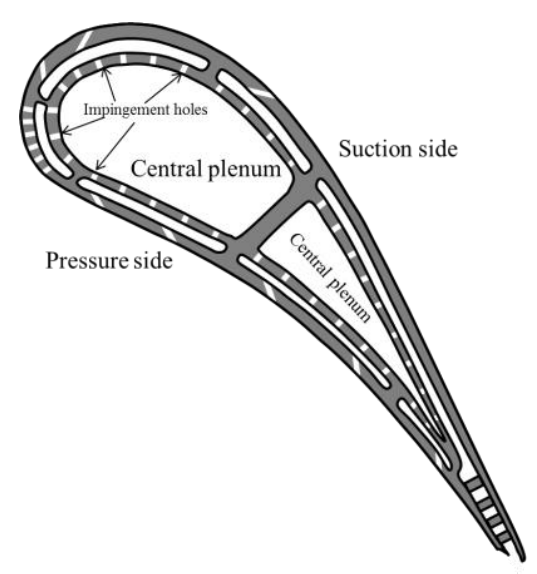

Fig. 1 Double wall cooling vane with short film cooling holes.

Early investigations on the turbine film cooling performance have been conducted by Drost et al. [8], Ames 
et al. [9], Cutbirth et al. [10, 11], and Sargison et al. [12]. Drost et al. [8] employed the transient liquid crystal technique to investigate the film cooling effectiveness and heat transfer coefficient on a turbine NGV airfoil. Tests were conducted for blowing ratios of 0.25 to 2.3 on the suction side, and 0.55 to 7.3 on the pressure side, with a density ratio of 1.65. It was reported that the increased mainstream turbulence had a weak influence on the suction side film cooling, and caused higher film cooling effectiveness on the pressure side. The effects of mainstream Mach and Reynolds number were attributed to changes of boundary layer thickness and flow acceleration. Ames et al. [9] investigated the influence of turbulence on vane film cooling distributions in a four-vane subsonic cascade. The Vane film cooling effectiveness distributions were documented in the presence of a low level of turbulence (1 percent) and were used to contrast results taken at a high level (12 percent) of large-scale turbulence. It was reported that the turbulence had a moderate influence on suction surface film cooling but had a dramatic influence on pressure surface film cooling, particularly at the lower velocity ratios. It was also found that the strong pressure gradients on the pressure surface of the vane altered film cooling distributions substantially. Sargison et al. [12] performed a comparison of adiabatic effectiveness performance of fan-shaped holes, converging slot, and cylindrical holes on a transonic nozzle guide vane in an annular cascade. Surface heat transfer coefficient, the adiabatic cooling effectiveness, and the derived net heat flux reduction were obtained. It was reported that the performance of fan-shaped holes was similar to the converging slot while they were both higher when compared to cylindrical holes in terms of adiabatic effectiveness and net heat flux reduction.

Guo et al. [13] employed the thin-film technique to investigate the effect of hole shape on the adiabatic film effectiveness on the suction side and pressure side in a fully cooled nozzle guide vane in a annular cascade. They found that, on the pressure side, the fan-shaped hole produced higher adiabatic film cooling effectiveness downstream of the cooling hole in comparison to the cylindrical hole, but at a faster decay as the flow developed downstream. Colban et al. [14] measured the adiabatic film effectiveness of fanshaped holes on the suction and pressure surfaces of a turbine vane in the presence or absence of showerhead film cooling. Their study showed that the severe lift-off that was observed for a single row, on the pressure and the suction side, was reduced in the presence of an upstream showerhead. Mhetras et al. [15] examined the effect of showerhead injection at the leading edge and the presence of compound angle holes on the pressure and suction sides of a fully cooled high pressure turbine blade in a stationary, linear cascade. The full-coverage film cooling consisted of six rows of compound angle shaped holes on the pressure side and four rows of such holes on the suction side. It was reported that higher blowing ratios provided higher effectiveness levels on the pressure side, while only marginal influence on the suction side film cooling effectiveness. It was also pointed that the secondary flow vortices such as the passage and the tip vortex significantly impacted the suction side film-cooling effectiveness distribution.

The suction side vane film cooling for shaped holes was investigated by Zhang et al. [16] by using the pressure sensitive paint technique. They found that adiabatic effectiveness increased for fan-shaped holes up to blowing ratio 1.5. Furthermore, Zhang et al. [17] also showed that adiabatic effectiveness decreased for fan-shaped holes on the pressure side if blowing ratio increased above 1.5. Nathan et al. [18] investigated the showerhead and one additional row of cooling on the suction and the pressure surface of a turbine vane in terms of overall cooling effectiveness. The hot spots on the surface within the showerhead region, which might be causes of failure for the turbine vane, were reported in their study. Recently, Nadali et al. [19] employed transient infrared thermography method to investigate the film cooling performance of cylindrical and fan-shaped holes on a cooled turbine guide vane. They presented the adiabatic film effectiveness and net heat flux reduction results due to coolant injection through double and multiple rows in the presence and absence of an upstream showerhead. They found that the choice of best cooling hole shape for film-cooling design was highly influenced by the number of cooling rows to be used and also the presence (or absence) of showerhead cooling.

The effect of full coverage film cooling and heat transfer on turbine blade has been investigated by several studies, including $[20,21]$. To meet the cooling demand, the turbine blades are more likely to utilize the full-coverage film 
cooling to achieve high cooling efficiency. Airfoils like double wall blades may have thin outer sheet of metal and use full-coverage film cooling with film cooling holes with short length-to-diameter ratios. Therefore, the present study is different from previous full-coverage film cooling studies because new experimental data are provided for fullcoverage turbine vanes with varied wall thickness and hole shape. Also data are presented to provide a detailed analysis of curvature effect on the film cooling performance in comparison to flat-plate data.

\section{Experimental Facilities}

\subsection{Five-Vane Linear Cascade}

A schematic of the test section is shown in Fig. 2, consisting of the cascade, open coolant loop, and the data acquisition system [22]. The inlet cross section of the test section is $450 \mathrm{~mm}$ (width) $\times 105 \mathrm{~mm}$ (height) and the exit cross section is $125 \mathrm{~mm}$ (width) $\times 105 \mathrm{~mm}$ (height). The mainstream is supplied by a centrifugal compressor and goes through a honeycomb-rectifier, which breaks larger vortices and makes the flow more uniform. The approaching flow conditions, including flow velocity, temperature, and turbulence intensity, are measured through thermocouples, five-hole probe, and hot-wire probe, which locate $5 C_{a x}$ upstream the vanes. The freestream turbulence intensity is measured to be $3.5 \%$ by a constant temperature anemometer system (DANTEC90N10) with hot-wire probe from Dantec Inc. Table 1 lists some flow conditions, with the inlet Reynolds number of $1.35 \times 10^{5}$ and the inlet Mach number of 0.065 . The operation conditions of the linear cascade is lower than the typical flow conditions of industrial gas turbine. However, the present study can provide reasonable estimation on the effects of turbine vane thickness and hole shape. Similar studies can be found in references $[10,11,14]$. Five turbine vanes are implemented to ensure periodicity in flow passages, and the center vane is the test model. Images of the test surface were captured through this acrylic interface by a CCD camera mounted outside the test section.

For the secondary flow supplement, an air compressor and high pressure gas tank is used to provide the coolant gas, i.e. air and $\mathrm{CO}_{2}$, respectively. The coolant massflow rate is controlled and measured by electric mass flowmeters. The coolant temperature is adjusted by a heat exchanger prior to entering the mainstream and a difference within $0.5^{\circ} \mathrm{C}$ to mainstream temperature is ensured due to pressure sensitive paint requirement. The coolant temperature in the cavity is measured by thermocouples.

In the experiments, blowing ratios of $\mathrm{M}=0.5,1.0$, and 1.5 are selected for the showerhead film cooling and pressure side film cooling. Blowing ratios of $\mathrm{M}=0.25,0.5$, and 1.0 are selected for the suction side film cooling. The density ratio for all the cases is 1.5 . The massflow rate corresponding to each passage is calculated by the following expression

$$
\dot{m}_{c}=\sum_{r=1}^{n} M \cdot\left[m_{m}\right]_{r} \cdot\left[A_{c}\right]_{r}
$$

where $\mathrm{r}$ is the hole row, $\mathrm{n}$ is the number of rows sharing one common passage, $m_{m}$ is the mainstream mass flux over row $\mathrm{r}$, and $A_{c}$ is the total area of all coolant holes in row $\mathrm{r}$. The mainstream mass flux $\left(m_{m}=\rho_{m} V_{m}\right)$ over row $\mathrm{r}$ is calculated from the local pressure information obtained by pressure measurement system.

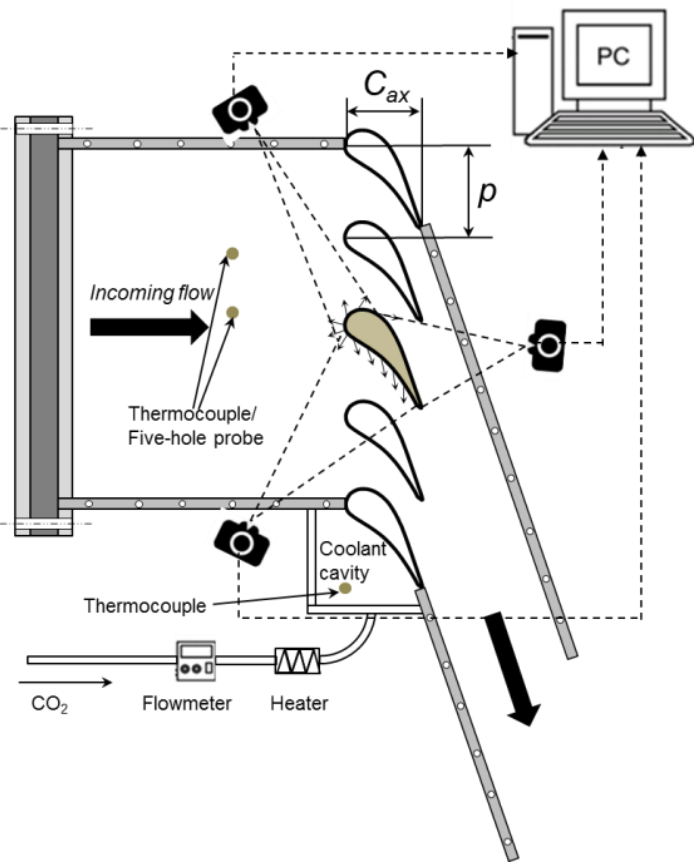

Fig. 2 Schematic of experimental test section. Table 1 Vane parameters and operation conditions

\begin{tabular}{ll}
\hline \hline Parameter & Value \\
\hline$C_{a x}(\mathrm{~m})$ & 0.0785 \\
$p / C_{a x}$ & 1.31 \\
$H / C_{a x}$ & 1.45 \\
$\mathrm{Re}_{\text {inlet }}$ & $1.35 \times 10^{5}$ \\
$\mathrm{Ma}_{\text {inlet }}$ & 0.065 \\
Inlet flow angle $\left(^{\circ}\right)$ & 0 \\
Exit flow angle $\left(^{\circ}\right)$ & 73 \\
\hline \hline
\end{tabular}




\subsection{Test Vane Details}

The vane coolant passages and hole locations are displayed in Fig. 3. The vane height is $0.114 \mathrm{~m}$ and the axial chord length is $0.0785 \mathrm{~m}$. The pitch length between two adjacent vanes is $0.103 \mathrm{~m}$. The leading edge of the test vane has six rows of showerhead film cooling holes. The true surface distances of the holes from the stagnation line are $\mathrm{S} / \mathrm{D}=-4.2,-1.8,0.9,3.6,6.0$, and 9.1, where negative $\mathrm{S} / \mathrm{D}$ values indicate suction side positions and positive $S / D$ values indicate pressure side positions. The stagnation line locates between the second and third rows of showerhead holes on the leading edge. Each showerhead row has 22 holes with a hole spacing of $4.5 \mathrm{~mm}(\mathrm{~s} / \mathrm{D}=4.5, \mathrm{D}=1 \mathrm{~mm})$ and a length-to-diameter (L/D) of 4.6. The showerhead hole has an inclination angle of $35^{\circ}$ and a compound angle of $90^{\circ}$. The six leading edge rows are staggered with respect to one another and the adjacent rows of holes inject into the freestream at opposite directions. Five rows of holes on the pressure side locate at true surface distances: $\mathrm{S} / \mathrm{D}=24(\mathrm{PS} 1$, 22 holes), 37 (PS2, 23 holes), 58 (PS3, 22 holes), 78 (PS4, 23 holes), and 102 (PS5, 22 holes). Three rows of holes on the suction side locate at true surface distances: $\mathrm{S} / \mathrm{D}=-14$ (SS1, 22 holes), -24 (SS2, 23 holes), -65 (SS3, 22 holes). Holes on pressure side or suction side have a hole spacing of $5.0 \mathrm{~mm}(\mathrm{~s} / \mathrm{D}=5, \mathrm{D}=1 \mathrm{~mm})$ and a length-to-diameter (L/D) of 5.2. Adjacent rows of holes on the pressure side or suction side are staggered with respect to one another. The coolant is delivered from seven passages built along the span of the vane. The massflow rate of each passage is controlled by the flowmeter. The six rows of showerhead film holes share a common passage, Pass 1. PS1 and PS2 rows share the next passage, Pass 2. SS1 and SS2 rows share a common passage, Pass 6 . The remaining rows of holes are supplied by separate passages.

Figure 4 shows geometry details of the holes employed on the suction side and pressure side. Table 2 lists the hole L/D values of thick and thin vanes. Figure $4 \mathrm{a}$ indicates the simple angle hole, which has an inclination angle of $35^{\circ}$ and hole L/D value around 2 for thin vane and 5 for thick vane. Figure $4 \mathrm{~b}$ indicates the compound angle hole, which has an inclination angle of $35^{\circ}$ and a compound angle hole of $45^{\circ}$. Figure $4 \mathrm{c}$ indicates the fan-shaped angle hole, which has an inclination angle of $35^{\circ}$ and lateral expansion angle of $10^{\circ}$. The length ratio of cylindrical portion to shaped portion is
$1 / 2$.
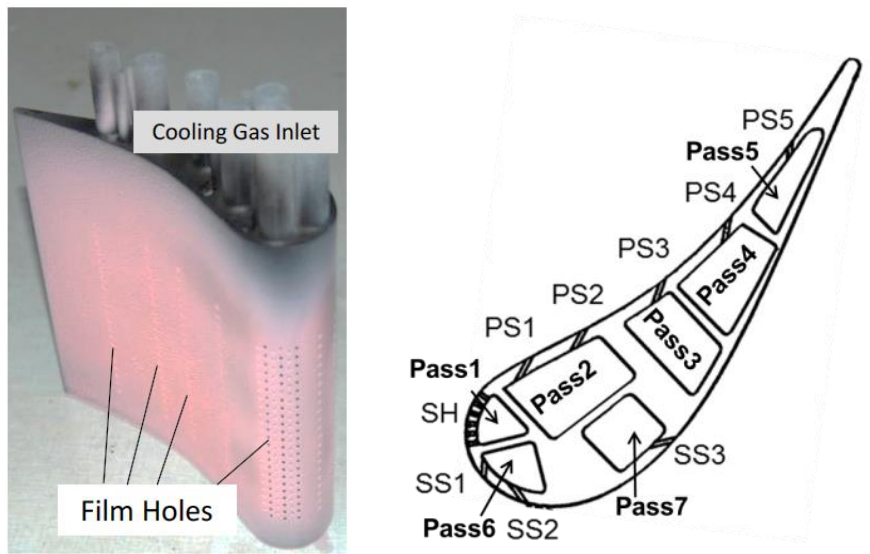

Fig. 3 Vane coolant passages and hole locations.

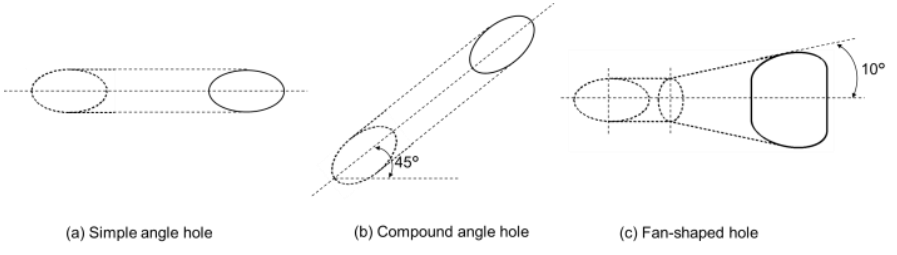

Fig. 4 Definition of hole shape and orientations: (a) simple angle hole, (b) compound angle hole, and (c) fan-shaped hole.

Table 2 Hole L/D values of thick and thin vanes

\begin{tabular}{cccc}
\hline \hline Thick Vane & L/D & Thin Vane & L/D \\
\hline SH & 4.6 & SH & 1.9 \\
PS1 & 5.2 & PS1 & 2.2 \\
PS2 & 5.1 & PS2 & 2.1 \\
PS3 & 5.2 & PS3 & 2.2 \\
PS4 & 5.0 & PS4 & 2.1 \\
PS5 & 2.9 & PS5 & 2.1 \\
SS1 & 3.1 & SS1 & 1.7 \\
SS2 & 5.0 & SS2 & 2.1 \\
SS3 & 5.0 & SS3 & 2.1 \\
\hline \hline
\end{tabular}

\section{Measurement Procedure}

3.1 Vane Surface Pressure Measurement and The Local Blowing Ratio

The Local blowing ratio is determined by the local mainstream mass flow and the coolant massflow rate. It is defined in the following expression

$$
M=\left(\frac{\rho_{c} V_{c}}{\rho_{\infty} V_{\infty}}\right)_{\text {local }}
$$


where $\rho$ and $V$ indicate flow density and velocity, while $\infty$ and $\mathrm{c}$ represent the parameters of mainstream and coolant, respectively. Before the cascade film cooling experiments, the surface pressure along the vane surface is obtained by the pressure measurement system, which is shown in Fig. 5. The surface pressure along the vane surface is recorded by the computer connected with the pressure transducers.

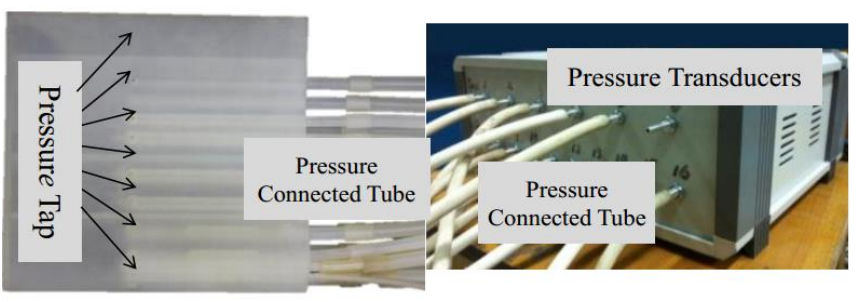

Fig. 5 Vane pressure measurement system.

Figure 6 shows the measured pressure data on the tested vane surface, which is also validated by the CFD simulation data. Based on the vane surface static pressure, the stagnation point total pressure, the inlet total temperature, the isentropic Mach number and the total temperature along the vane surface can be deduced by the equation

$$
\begin{gathered}
M a=\sqrt{\frac{2}{k-1}\left[\left(\frac{P}{P^{*}}\right)^{-(k-1) / k}-1\right]} \\
\frac{T^{*}}{T}=1+\frac{k-1}{2} M a^{2}
\end{gathered}
$$

Combining with measured coolant massflow rate, the local blowing ratio of every row of holes can be determined form Eqn. (2).

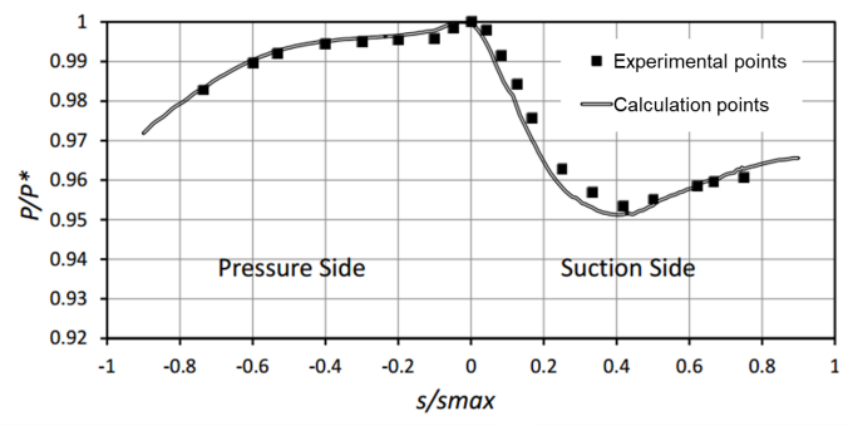

Fig. 6 Vane surface pressure distribution.

\subsection{Pressure Sensitive Paint and Adiabatic}

\section{Effectiveness}

Pressure sensitive paint (PSP) has been widely used in measuring film cooling effectiveness such as Russin et al. [23] and Ahn et al. [24]. Recently detailed uncertainty analysis work has been conducted by Natsui et al. [25]. It is proved that the PSP technology is a powerful tool for film cooling effectiveness measurements due to its high precision, stability, and repeatability.

Film cooling effectiveness measured using PSP is based on heat/mass analogy and thus can be expressed by oxygen concentration as [23]:

$$
\eta=\frac{T_{\infty}-T_{m i x}}{T_{\infty}-T_{c}} \rightarrow \eta=\frac{C_{\infty}-C_{m i x}}{C_{\infty}-C_{c}}
$$

where $C_{\infty}$ is the oxygen mass concentration of mainstream gas, $C_{c}$ is the oxygen mass concentration of coolant gas, and $C_{m i x}$ is the oxygen mass concentration of the gas mixture on the test surface. Oxygen mass concentration can be converted to oxygen partial pressure and the expression of film cooling effectiveness is reorganized as:

$$
\eta=1-\frac{1}{\left[\frac{\left(P_{O 2}\right)_{a i r}}{\left(P_{O 2}\right)_{\text {mix }}}-1\right] \frac{M_{c}}{M_{\text {air }}}+1}
$$

in which the $\left(\mathrm{P}_{\mathrm{O} 2}\right)_{\text {air }}$ is the oxygen partial pressure of mainstream gas, $\left(P_{O 2}\right)_{m i x}$ is the oxygen partial pressure of coolant gas, $\mathrm{M}_{\mathrm{c}}$ is the average molecular weight of air, and $\mathrm{M}_{\text {air }}$ is the molecular weight of coolant.

PSP is an oxygen-quenching photo-luminescent material which emits light when excited. The emitted light intensity is reduced with the existence of oxygen. Currently, the UniFIB PSP (UF470-750) from ISSI Inc. is used in the experiments. LED light with a wavelength of $470 \mathrm{~nm}$ excites the PSP and the emitted light is recorded by a CCD camera with a filer to shield the excitation light [26]. Four different kinds of images are recorded during the experiments: image with mainflow and $\mathrm{N}_{2} / \mathrm{CO}_{2}$, image with mainflow and air, image without mainflow and coolant (reference image), and background image (to remove noise). The light intensity is extracted from these images and normalized in a manner to get light intensity ratio. The light intensity ratio is converted to pressure ratio using a calibration curve shown in Fig. 7 which was obtained using a calibration device similar to $\mathrm{Li}$ et al. [26]. The pressure was normalized with the room pressure $\left(\mathrm{P}_{0}\right)$, while the intensity data were normalized with three different reference conditions recorded at room 
pressure and at the same temperature of the correspondent curve $\left(I_{0}\right)$.

The typical uncertainty of adiabatic film cooling effectiveness measurements varies with different coolant gas, i.e. $\mathrm{CO}_{2}$. The uncertainty is estimated to be $6 \%$ for $\eta$ higher than 0.3 , and $11 \%$ for $\eta$ around 0.1 [26]. All the uncertainty analysis is based on $95 \%$ confidence.

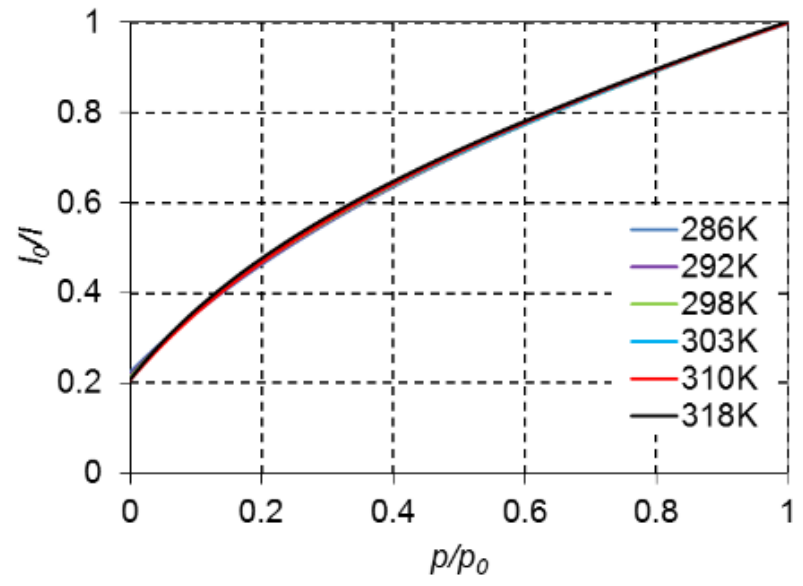

Fig. 7 PSP calibration curves under different temperature conditions

\section{Results and Discussion}

\subsection{Experimental Validation}

In order to validate the measurement technique, the experimental measured film cooling effectiveness on the flat-plate is compared with previous studies. Figure 8 shows the laterally averaged film cooling effectiveness comparison between present data and published data for simple angle hole and fan-shaped hole. Figure 8a shows the comparison of simple angle hole with the data from Waye et al. [27] and Saumweber et al. [28]. The pitch-to-diameter ratio, lengthto-diameter ratio, and density ratio in the published data are quite similar to the present study. Some small differences are primarily attributed to inlet boundary conditions, like turbulence intensity and boundary layer state. Figure $8 \mathrm{~b}$ shows the comparison of fan-shaped angle hole with data from Saumweber et al. [28], An et al. [29], and Dittmar et al. [30]. Overall, it can be stated that the present film cooling effectiveness agree well with published data, which demonstrates the high quality of the PSP measurement technique.
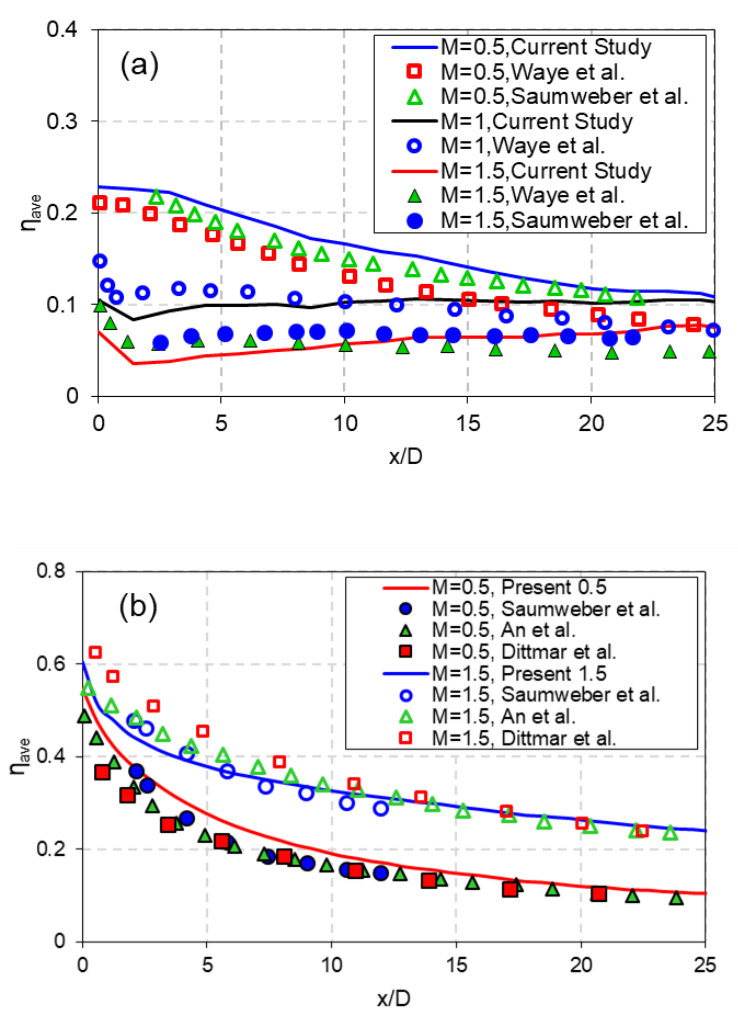

Fig. 8 Laterally averaged film cooling effectiveness comparison with published data: (a) simple angle hole, (b) fan-shaped hole.

\subsection{Showerhead Film Cooling Results}

The showerhead film cooling effectiveness distribution is shown in Fig. 9 at three blowing ratios of $\mathrm{M}=0.5,1.0$, and 1.5. In the stagnation region near $\mathrm{S} / \mathrm{D}=0$, the internal-toexternal pressure difference is low, resulting in low jet momentum in the three rows of holes. Also low freestream momentum causes weak jet-to-crossflow interaction, which leads to less deflected jet trajectory than the other rows of holes. As the flow develops downstream, the deflected jet trajectory is evidently observed and the film cooling effectiveness is remarkably improved due to more coolant coverage. More coolant coverage in the downstream region is caused by coolant accumulations from upstream hole rows, and increased jet momentum due to larger internal-toexternal pressure difference.

As the blowing ratio increases from $M=0.5$ to $M=1.5$, the variation trends for stagnation region and downstream region are opposite. For the stagnation region, the increase of blowing ratio leads to increased film cooling effectiveness near the hole exit or two adjacent film rows. 
This is because more coolant accumulates near the stagnation region as the blowing ratio increases. However, the trend is reversed for downstream regions where $S / D \leq 5$ and $S / D \geq 5$. Increasing the blowing ratio generally brings about a visible drop in film cooling effectiveness, which is consistent with the data of Gao et al. [31]. It is suspected that the increased blowing ratio causes the jet to be more susceptible to lift off the surface and decreases the film cooling effectiveness.
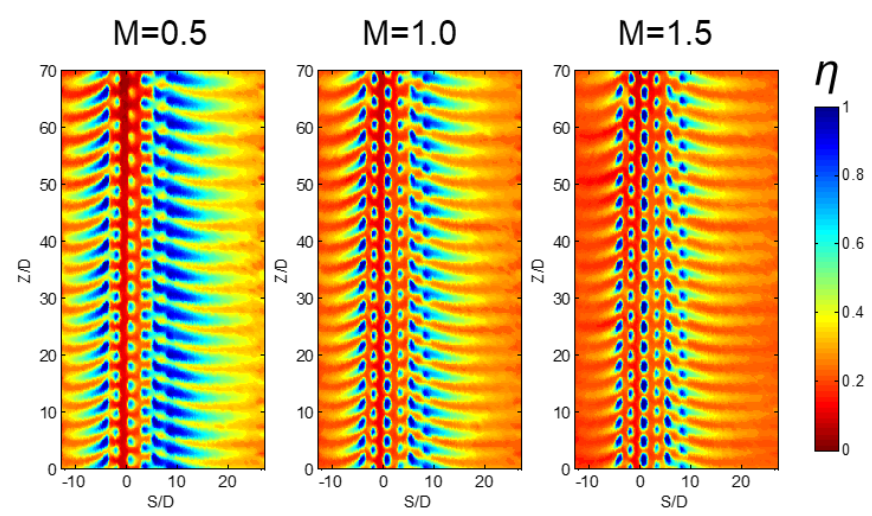

Fig. 9 Contours of showerhead film cooling effectiveness at three blowing ratios.

Figure 10 shows the laterally averaged film cooling effectiveness with varied hole $\mathrm{L} / \mathrm{D}$ at blowing ratios of $M=0.5$ and $M=1.5$. The figure shows the effect of varying wall thickness on the showerhead film cooling effectiveness. Figure 10a shows that the film cooling effectiveness in the stagnation region is remarkably lower than the downstream region due to lower coolant coverage. At region $S / D \geq 0$, i.e., from stagnation line to pressure side, increasing the wall thickness produces slight improvement in film cooling effectiveness. At region $\mathrm{S} / \mathrm{D} \leq 0$, i.e., from stagnation line to suction side, the trend is reversed and increasing the wall thickness decreases the film cooling effectiveness slightly. As the blowing ratio is increased to $M=1.5$ in Fig. 10b, the film cooling effectiveness in the stagnation region shows noticeable improvement and is comparable to the downstream region, which is ascribed to more cooling injection. A closer inspection to the figure indicates a slight decrease in film cooling effectiveness caused by the increase in wall thickness. It is concluded that the dependency of showerhead film cooling effectiveness on reducing wall thickness is associated with blowing ratios. It is also found that the variation of showerhead film cooling effectiveness due to wall thickness reduction is generally marginal. This may indicate that the showerhead overall cooling efficiency can be enhanced through reducing wall thickness and wall thermal resistance, with limited influence on the film cooling effectiveness.
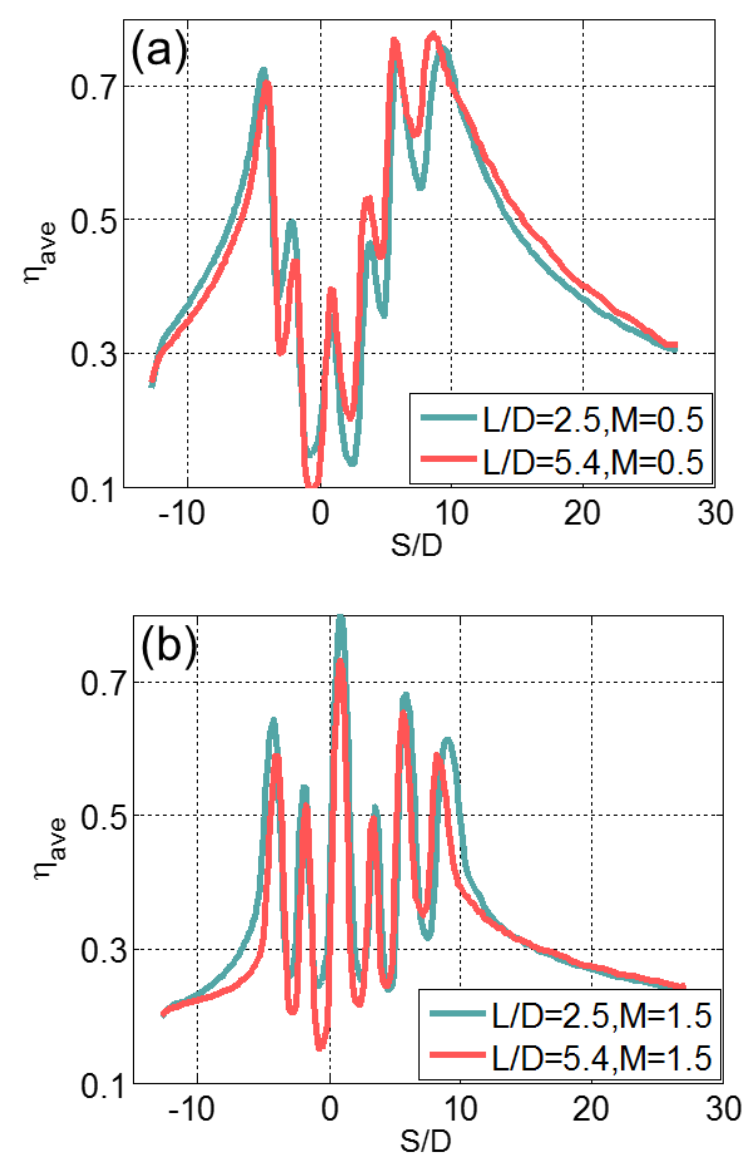

Fig. 10 Laterally averaged film cooling effectiveness with varied hole $L / D$, (a) $M=0.5$, (b) $M=1.5$.

\subsection{Suction Side Film Cooling Results}

The blowing ratios for the hole rows of SS1, SS2, and SS3 range from $\mathrm{M}=0.25$ to $\mathrm{M}=1.0$. Blowing ratios for individual hole row is controlled and identical to other hole rows for better comparison.

\subsubsection{Hole shape variation effect}

Figure 11 shows the contours of suction side film cooling with varied hole shape at the blowing ratio of $M=0.5$. Figure 11a shows the film cooling effectiveness distribution of simple angle hole. It is observed that the long and welldefined coolant traces are produced behind SS1-SS3 rows due to strong flow acceleration. For example, the coolant traces travel around 55 hole diameters downstream from SS2 row. The coolant from SS1 row extends to downstream of SS2 row, which leads to accumulated coolant coverage 
and elevated film effectiveness. Also observed is that the coolant traces become thinner and shorter downstream SS3 row compared to SS2 row, which may be an indicative of coolant lift-off and higher mixing losses. Figure 11b displays the film cooling contour of compound angle hole. Higher film cooling effectiveness is observed downstream SS1 and SS2 rows, since the compound angle favorably suppresses the jet into the surface. The coolant traces downstream SS1 and SS2 rows are less slender and more diffused in comparison to the simple angle hole. The jet trajectory of SS3 behaves quite identically to the simple angle hole due to high freestream acceleration and convex curvature. Figure 10c shows that fan-shaped hole brings about significant improvement in film cooling effectiveness in comparison to simple and compound angle holes. The coolant traces become fuller and more diffused in lateral directions. The shaped portion expansion reduces the jet momentum and the jets stay closer to the surface. The decreased jet-to-momentum interaction also reduces the coolant dispersion and diffusion, which results in higher film cooling effectiveness. The coolant accumulations from SS1 and SS2 rows produce high film coverage which extends around 40 hole diameters.

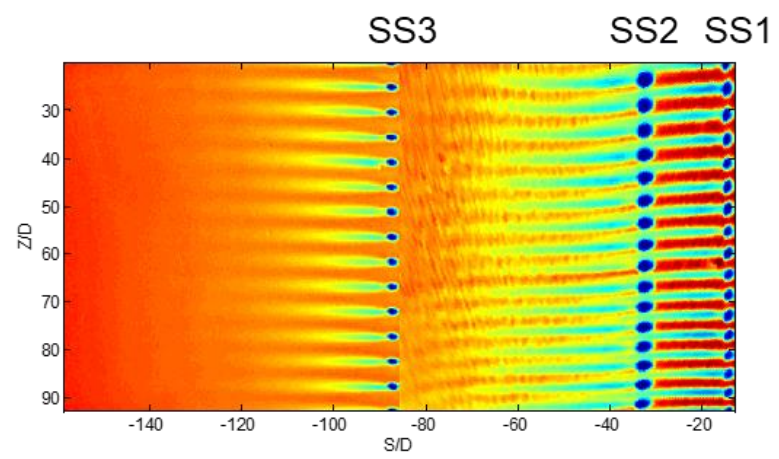

(a) Simple angle hole

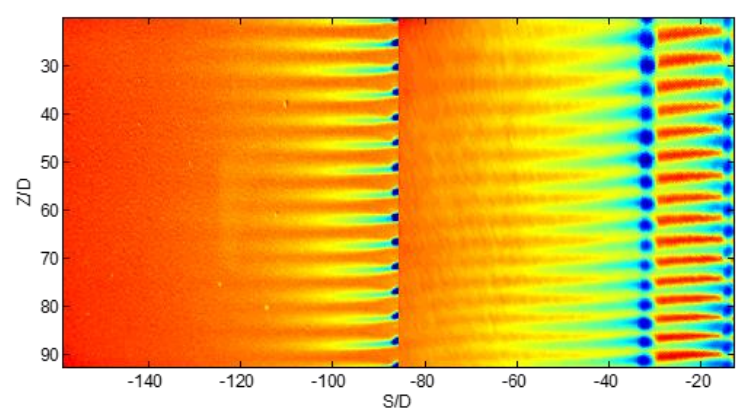

(b) Compound angle hole

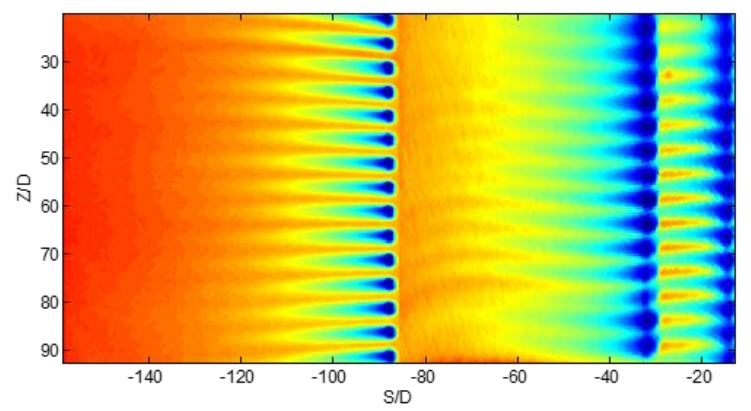

(c) Fan-shape hole

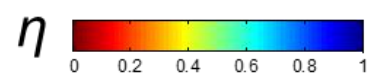

Fig. 11 Contours of suction side film cooling effectiveness with varied hole shape at $M=0.5$, (a) simple angle hole, (b) compound angle hole, and (c) fan-shaped hole.

A quantitative description of the hole shape effect on the film cooling effectiveness is plotted in Fig. 12. Data with blowing ratios of $\mathrm{M}=0.25$ and $\mathrm{M}=1.0$ are shown in terms of laterally averaged data. Figure 12a shows that the hole shape variation generally produces marginal influence on the laterally averaged film cooling effectiveness with the low blowing ratio, except regions downstream SS3 row. No discernable jet lift-off is observed for SS1 and SS2 rows due to low jet momentum and high flow acceleration. Fanshaped hole shows visible superiority over simple and compound angle holes in achieving higher film cooling effectiveness downstream SS3 row. This is ascribed to the jet lift-off occurred in simple and compound angle holes, which results in lower film cooling effectiveness. As the blowing ratio is increased to $M=1.0$ in Fig. $12 b$, the film cooling effectiveness is remarkably reduced for simple and compound angle holes from SS1 to SS3 rows due to jet liftoff. On the contrary, the fan-shaped hole shows remarkable improvement of film cooling effectiveness. The improvement is more pronounced between SS1 and SS3 rows than downstream SS3 row. The enlarged hole exit area suppresses the jet lift-off and provides better film coverage. Fan-shaped hole shows higher film cooling effectiveness up to $0.2-0.3$ than cylindrical holes downstream SS2 row. The superiority decreases downstream SS3 row due to coolant dispersion and diffusion. It is concluded that improvement in film cooling effectiveness due to hole shape variation becomes more pronounced as blowing ratio increases. 

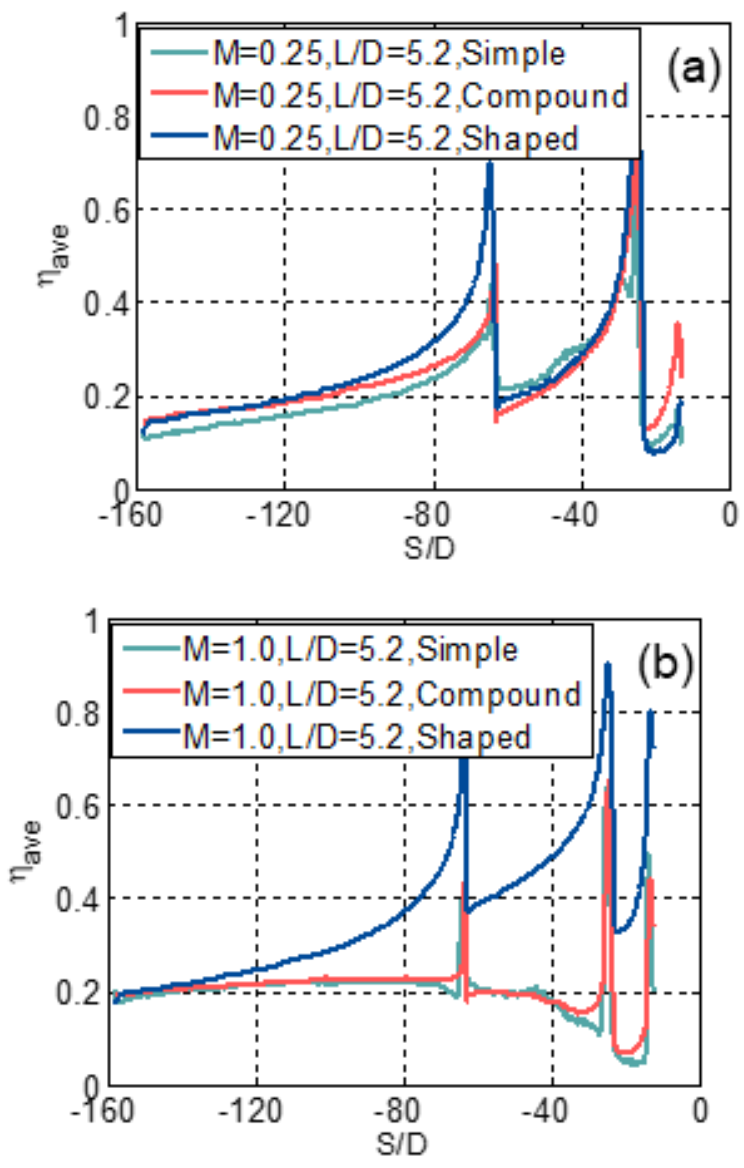

Fig. 12 Laterally averaged film cooling effectiveness with varied hole shape, (a) $M=0.25$, (b) $M=1.0$.

\subsubsection{Wall thickness effect}

The wall thickness effect on film cooling effectiveness is plotted in Fig. 13 at blowing ratio of $M=0.5$. The data of simple angle hole, compound angle hole, and fan-shaped hole are shown in Figs. 13a, 13b, and 13c, respectively. It can be stated that the wall thickness variation has very limited influence on the suction side film cooling effectiveness irrespective of hole shape. Only marginal improvement is observed between SS2 and SS3 rows in Fig. $13 \mathrm{c}$ as the wall thickness is reduced. The results on the suction side are not consistent with the flat-plate results obtained by Li et al. [5, 32]. The experiments in the flat-plate [5] and present study were conducted in the same wind tunnel and have almost similar hole shapes. The flat-plate film cooling results indicated that as the hole $\mathrm{L} / \mathrm{D}$ increases, the film cooling effectiveness increases for simple angle hole and fan-shaped hole, while remains almost invariant for the compound angle hole. This is associated with the in-hole vortical structures and mainstream kidney vortex development, which was revealed by the accompanying LES results [33]. Therefore, one can see that the variation trends of simple angle hole and fan-shaped hole in the suction side are inconsistent with the flat-plate results. This is suspected to be caused by the flow field features near the suction side, among which the cross-stream pressure gradient due to curvature dominates. The cross-stream pressure gradient causes bulk flow movement from pressure side to suction side and suppresses the coolant jet to the wall, which remarkably increases the film cooling effectiveness [35]. Strong flow acceleration occurs on the suction side due to reduced passage cross-sectional area, which causes local reduction in near-wall turbulence intensity. The convex curvature also favorably stabilize the flow and reduce nearwall turbulence intensity. The reduced near-wall turbulence favorably increases the film cooling effectiveness on the suction side, which is demonstrated by Narazary et al. [34]. The increase in film cooling effectiveness, which is caused by cross-stream pressure gradient and reduced near-wall turbulence, compensates with the decrease of film cooling effectiveness due to wall thickness reduction, for the simple angle hole and fan-shaped hole. Therefore, it is concluded that the film cooling results obtained on a flat-plate cannot be used for vane film cooling design without any modification. The limited influence on the suction side film cooling effectiveness caused by wall thickness reduction may suggest that overall cooling efficiency can be enhanced through reducing wall thickness and wall thermal resistance.

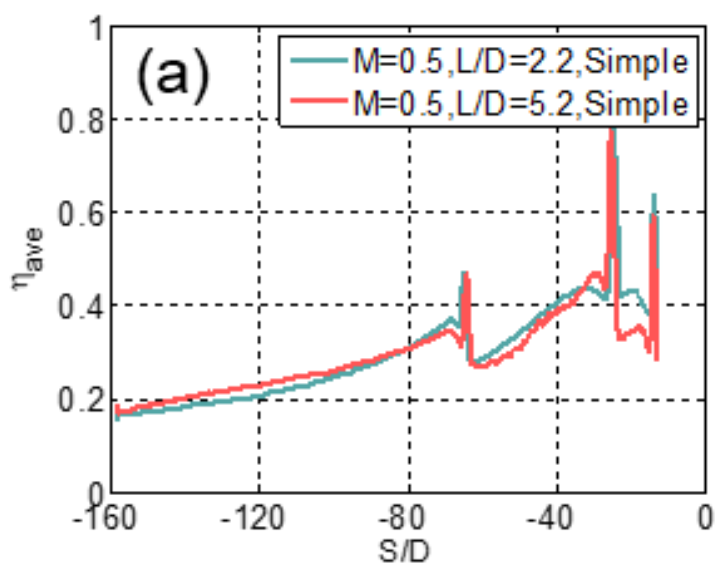



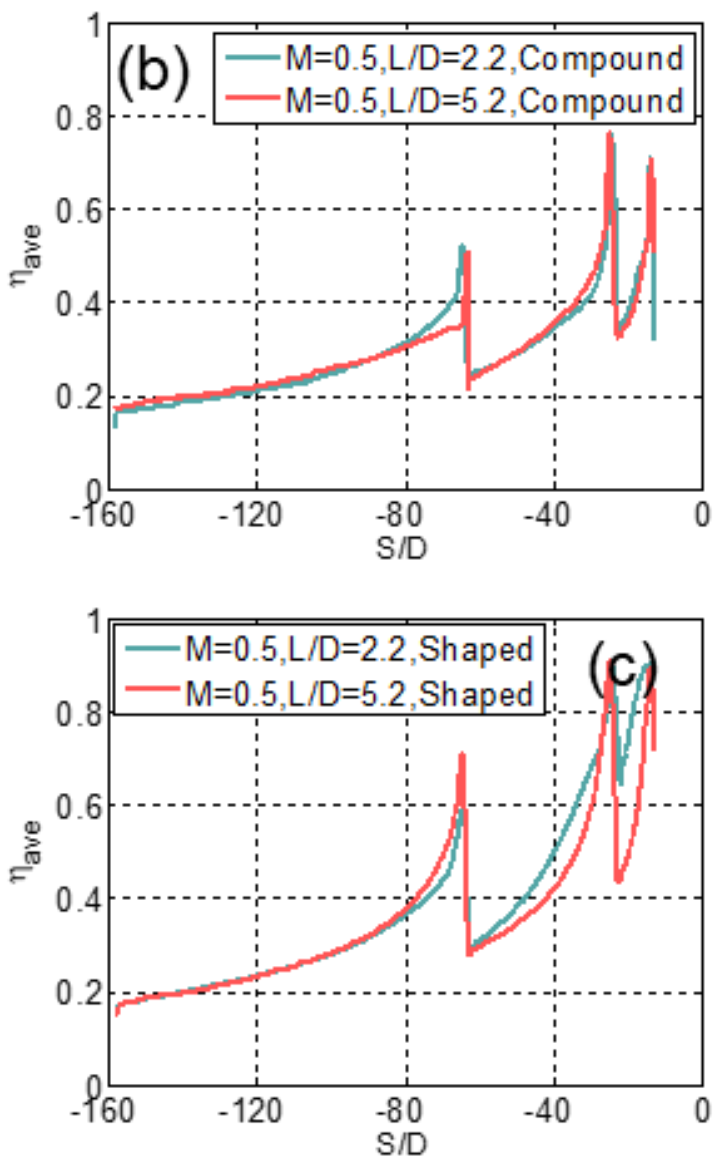

Fig. 13 Laterally averaged film cooling effectiveness with varied hole $L / D$ at $M=0.5$, (a) simple angle hole, (b) compound angle hole, and (c) fan-shaped hole.

\subsection{Pressure Side Film Cooling Results}

The blowing ratios for the hole rows of PS1 to PS5 range from $M=0.5$ to $M=1.5$. Blowing ratios for individual hole row is controlled and identical to other hole rows for better comparison.

\subsubsection{Hole shape variation effect}

Figure 14 shows the contours of pressure side film cooling effectiveness with varied hole shape at $M=0.5$. Overall, the simple angle hole shows a quite similar film cooling pattern to that of compound angle hole, except some local improvement near the hole trailing edge for the compound angle hole. This indicates that the compound angle hole shows rare superiority over simple angle hole in cascade conditions. However, the film cooling effectiveness is greatly improved due to the fan-shaped hole. It is observed that the coolant traces are smeared and shorter in comparison to these on the suction side, which is caused by the local high near-wall turbulence generated by concave curvature and a slower and thicker boundary layer. The concave curvature causes instability to the near-wall flow and increases the turbulence intensity. A closer inspection also reveals that the PS1 and PS2 rows show longer and fuller coolant traces, while PS3-PS5 rows show shorter and slender coolant traces. This is associated with local turbulence intensity and local freestream acceleration. The local turbulence is gradually intensified due to concave curvature instabilization effect as the flow develops downstream, which promotes jet dispersion and turbulence diffusion. Also strong freestream acceleration occurs as the flow approaches the throat or trailing edge of pressure side, which strengthens the vorticity of kidney vortex [15]. The accelerating boundary layer also promotes the attachment of kidney vortex to the wall [15]. These flow features contribute to the film cooling effectiveness degradation from leading to trailing edge on the pressure surface.

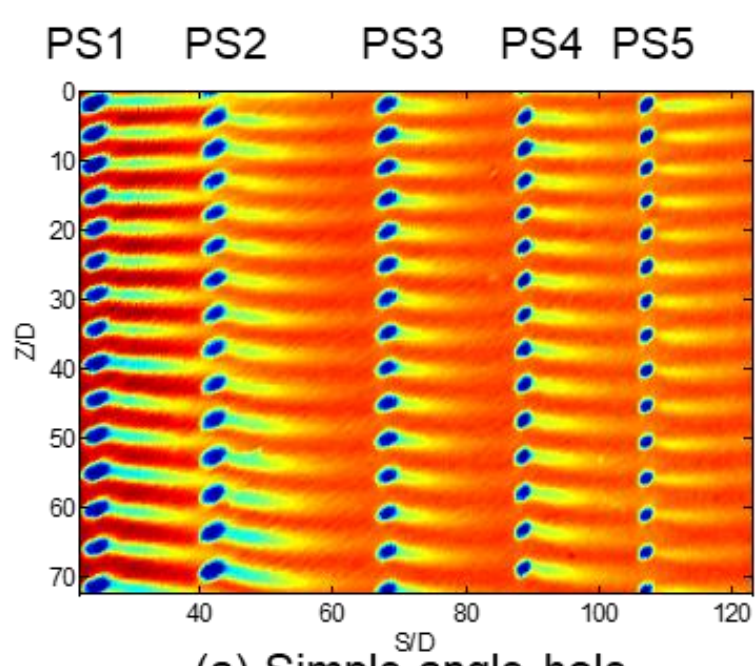

(a) Simple angle hole

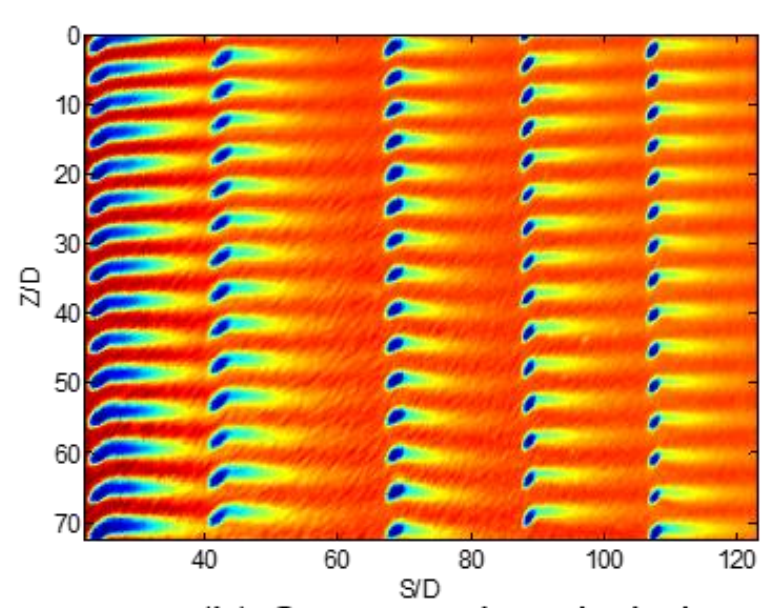

(b) Compound angle hole 


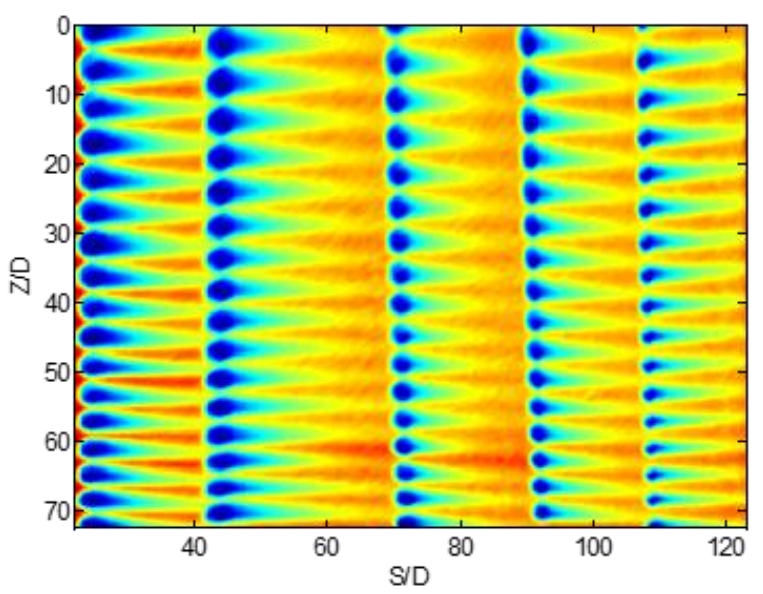

(c) Fan-shaped hole

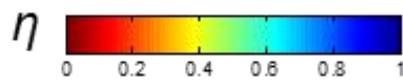

Fig. 14 Contours of pressure side film cooling effectiveness with varied hole shape at $M=0.5$, (a) simple angle hole, (b) compound angle hole, and (c) fan-shaped hole.

The laterally averaged film cooling effectiveness plot as a function of hole shape is shown in Fig. 15 at blowing ratios of $\mathrm{M}=0.5$ and $\mathrm{M}=1.5$. It is evidently observed that the fanshaped hole shows much higher film cooling effectiveness than the cylindrical holes, and the superiority becomes more pronounced as the blowing ratio increases. This is due to jet lift-off occurring for the cylindrical holes at high blowing ratios. It is also noticed that the simple and compound angle holes show quite identical film cooling effectiveness distributions on the pressure side surface. This variation trend on the pressure surface is consistent with that on the suction surface.

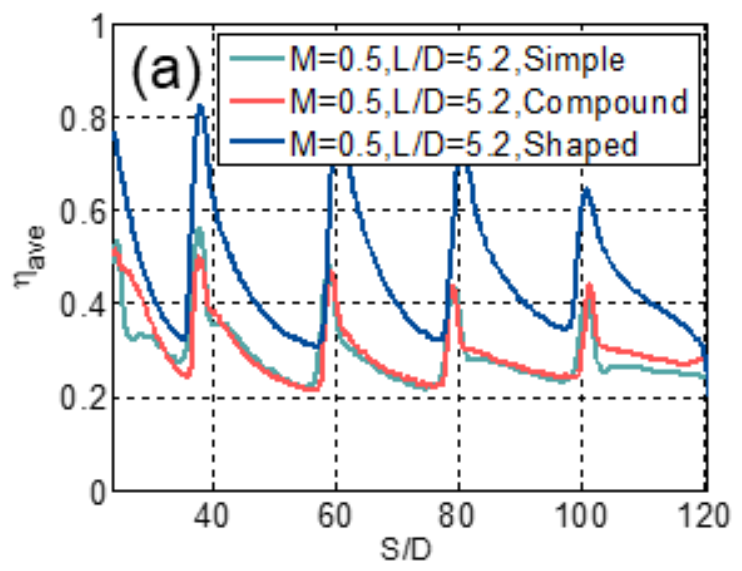

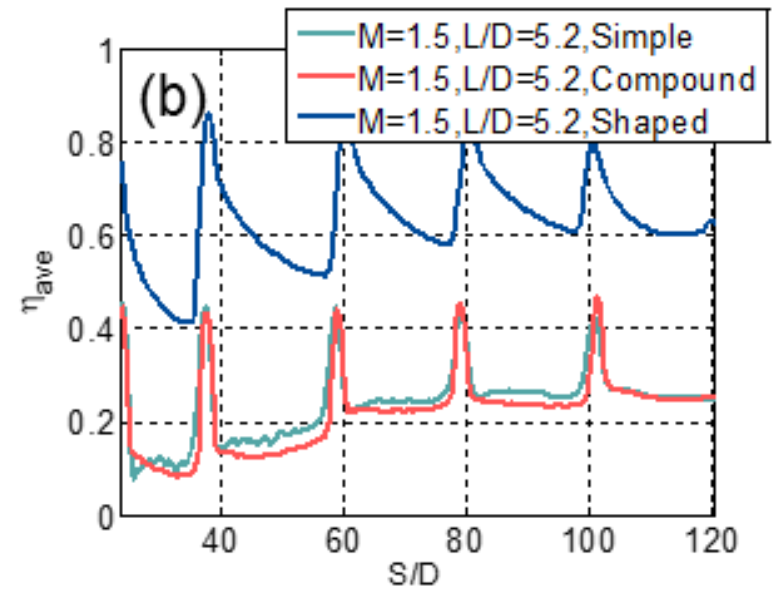

Fig. 15 Laterally averaged film cooling effectiveness with varied hole shape, (a) $M=0.5$, (b) $M=1.5$.

\subsubsection{Wall thickness effect}

The laterally averaged film cooling effectiveness of cylindrical and fan-shaped holes is shown in Fig. 16 to reflect the wall thickness effect. Figure 16a shows that the film cooling effectiveness of simple angle hole increases as the hole $\mathrm{L} / \mathrm{D}$ increases, which is consistent with the flat-plate results [5]. Increased hole L/D allows a more complete degradation of hole velocity deficit and attenuated in-hole counter rotating vortex pair, which promotes the coolant attachment to the wall and increases the film cooling effectiveness. Figure $16 \mathrm{~b}$ shows that increasing the hole L/D provides marginal influence on the film cooling effectiveness of compound angle hole, which is also reflected by the flat-plate results [5]. The accompany LES results indicated that the compound angle hole with short L/D generates additional shearing vortices to offset the single main rotating vortex and thus enhance film cooling performance [5]. Figure 16c shows that, for the fan-shaped hole, increasing the hole L/D produces remarkable improvement on the film cooling effectiveness, as illustrated by the flat-plate results [32]. The improvement is more pronounced than the simple angle hole. This is because increased hole L/D leads to more expanded hole exit area and more complete development of in-hole flow. The wall thickness effect on the pressure side film cooling is different from the suction side film cooling. Therefore, it is recommended that short fan-shaped hole with high cooling performance should be developed if designers want to make use of thin turbine airfoils. 

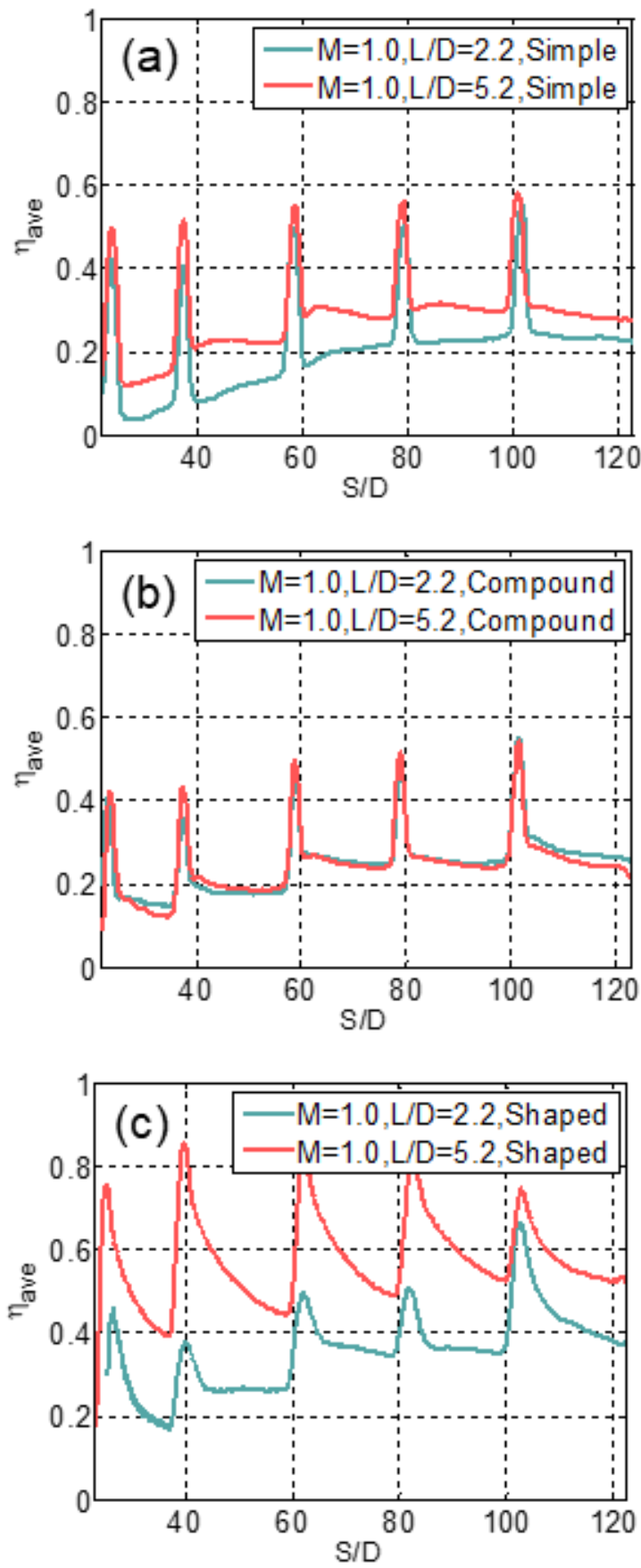

Fig. 16 Laterally averaged film cooling effectiveness with varied hole $L / D$ at $M=1.0$, (a) simple angle hole, (b) compound angle hole, and (c) fan-shaped hole.

\subsubsection{Superposition method validation}

Since the pressure side utilizes the full coverage film cooling, the superposition effect can be estimated through comparing the experimental data with the predictive data by Sellers' method [36]. The additive method proposed by Sellers is defined in the below equation:

$$
\eta(x)=\sum_{j=1}^{n} \eta_{j}(x) \prod_{i=0}^{j-1}\left(1-\eta_{i}(x)\right)
$$

Figure 17 shows the comparison of experimental data and predictive data on pressure side with varied hole shape and hole $\mathrm{L} / \mathrm{D}$ at blowing ratio of $\mathrm{M}=1.0$. The data used for prediction is the experimental data of single row hole at $M=1.0$. Evidently, the predictive data agree well with the experimental data, except for some local discrepancy occurs beyond $S / D=60$ at Fig. 17b. Generally, it might be stated that superposition effect of the full coverage film cooling on the vane pressure side, with the streamwise hole pitch around 20D, can be adequately predicted by the Sellers' method. The hole geometry variations, including hole shape and hole $\mathrm{L} / \mathrm{D}$, and the cascade flow conditions, like surface curvature, flow acceleration, and turbulence production and dispersion, exert limited influence on the application of the Sellers' method. 

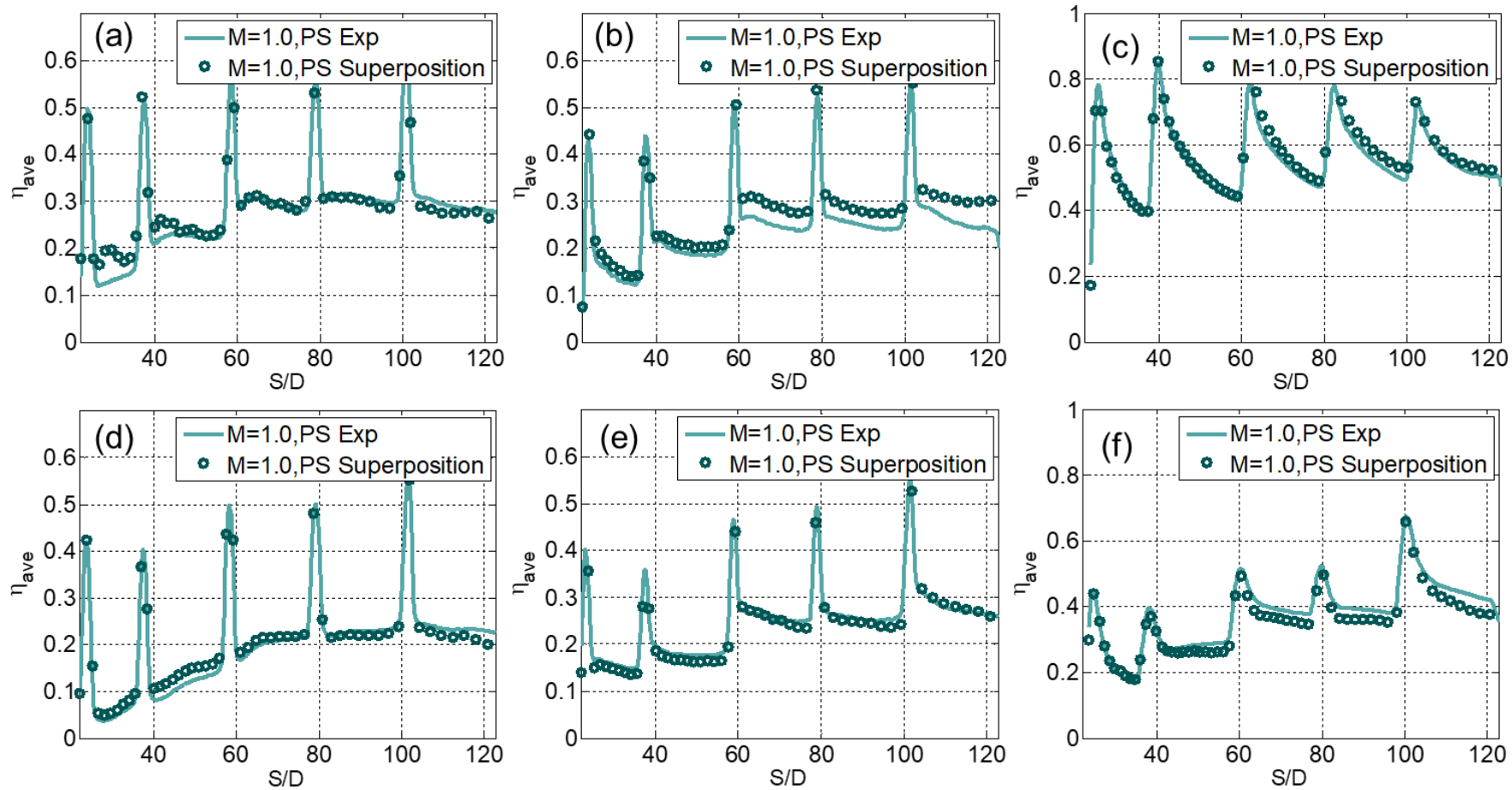

Fig. 17 Superposition method validation of laterally averaged film cooling effectiveness on pressure side with varied hole shape and hole $L / D$ at $M=1.0$, (a) simple angle hole-thick, (b) compound angle hole-thick, (c) fanshaped hole-thick, (d)simple angle hole-thin, (e) compound angle hole-thin, (f) fan-shaped hole-thin.

\subsection{Discussion on Surface Curvature Effect}

Surface curvature effect on film cooling effectiveness of cylindrical hole has been studied extensively, with convex curvature increasing film effectiveness and concave curvature decreasing film effectiveness. Generally, curvature effects have been presumed to scale with r/D at the film cooling hole location, where $r$ is the radius of curvature and D is coolant hole diameter. Previous studies indicated that, for the film jet on curve surface, the balance between the centrifugal force and the cross-stream pressure gradient was crucial for determining the jet trajectory [37, 38]. Ito et al. [37] deduced that if the tangential momentum of jets was less than unity, the cross-stream pressure gradient overwhelmed the centrifugal force and the jet came closer to a convex wall, but moved way from a concave wall, which resulted in increasing film cooling effectiveness on a convex wall while decreasing film cooling effectiveness on a concave wall. If the tangential momentum of jets was larger than unity, the centrifugal force overwhelmed the crossstream pressure gradient and the variation trend of film cooling effectiveness on convex or concave surface was reversed. Schwarz et al. [38] indicated that several factors influenced the film cooling performance of a row of holes on curve surface, including tangential momentum of jets, normal momentum of jets, concave instabilities, and crossstream pressure gradient. The tangential momentum of jets determines the balance between the centrifugal force and the cross-stream pressure gradient. The normal momentum of jets works to degrade film cooling effectiveness on concave or convex surface, since it tends to pull the jet away from the surface. The concave instabilities indicates high turbulence mixing on concave wall caused by the Gortler's vortices due to concave curvature. The cross-stream pressure gradient is pointing from a high pressure region near the concave surface to a low pressure region near the convex surface, which tends to move the film jet into a convex wall and away from a concave wall. The turbine blade generates concave curvature and convex curvature on the pressure side and suction side respectively, which produces opposite influence on the film cooling effectiveness. Therefore, the present study evaluates the blade surface curvature effect on the film cooling effectiveness of simple angle hole and fan-shaped hole with varied hole $\mathrm{L} / \mathrm{D}$, by comparing the vane data to the flat-plate data $[5,32]$. 
Figure 18 shows the comparison of laterally averaged film cooling effectiveness of simple angle hole with $\mathrm{L} / \mathrm{D}=5.2$ with flat-plate data. Data presented on the pressure side locate at PS3 and have a curvature of $r / D=99$, and on the suction side locate at SS3 and have a curvature of $r / D=90$. Figure 18a shows that, on the pressure side, the film cooling effectiveness is reduced for $M=0.5$, while elevated for $M=1.0$ and $M=1.5$, compared to the flat-plate data. This variation trend is consistent with the findings of Qin et al. [35]. Qin et al. [35] found that the concave curvature caused a reduction of film cooling effectiveness when the blowing ratio is less than 0.8 , while an increase when the blowing ratio is larger than 0.8 . The variation trend is ascribed to the relationship between the cross-stream pressure gradient and the centrifugal force, and the concave instability. At the blowing ratio of $\mathrm{M}=0.5$, the tangential momentum of jet is low, and then the cross-stream pressure gradient overwhelms the centrifugal force, which pulls the jet away from the concave surface and leads to reduced film cooling effectiveness in comparison with flat-plate data. On the other hand, the intensified turbulence intensity due to Gortler's vortices causes enhanced turbulence mixing and degrades the attachment of jet to wall, which results in the decrease of film cooling effectiveness. At medium to high blowing ratios, the tangential momentum of jet is high, which leads to an overwhelming centrifugal force over the cross-stream pressure gradient. The jet is pushed towards the concave surface and the film cooling effectiveness is promoted. Also, the high turbulence motion and turbulence mixing favorably promotes the coolant coverage on the surface when the jet detaches the surface and increases the film cooling effectiveness.

In Fig. 18b, for the suction side, the film cooling effectiveness on convex wall is higher than the flat-plate data at the blowing ratios of $\mathrm{M}=0.25$ and $\mathrm{M}=0.5$, while lower than the flat-plate data at the blowing ratio of $M=1.0$. This variation trend is also consistent with the findings of Qin et al. [35]. Qin et al. [35] found that the convex curvature enhances the film cooling effectiveness when the blowing ratio is less than 0.8 , while degrades the film cooling performance when the blowing ratio is larger than 0.8. This variation trend is mainly associated with the relationship between the centrifugal force and the crossstream pressure gradient, as indicated by Ito et al. [37]. At low blowing ratio of $\mathrm{M}=0.25$ and $\mathrm{M}=0.5$, the tangential momentum of jet is low, and so the force induced by the cross-stream pressure gradient is higher than the centrifugal force. This contributes to push the jet into the convex surface and enhances the film cooling effectiveness over the flatplate data. At the blowing ratio of $\mathrm{M}=1.0$, the force induced by the cross-stream pressure gradient is lower than the centrifugal force, and hence the jet is pulled away from the convex surface, resulting in reduced film cooling effectiveness compared with flat-plate data. On the convex surface, the mainstream is highly accelerated and the nearwall turbulence intensity is weakened. Therefore, the turbulence intensity plays a second-order role on the convex surface on influencing the film cooling effectiveness.
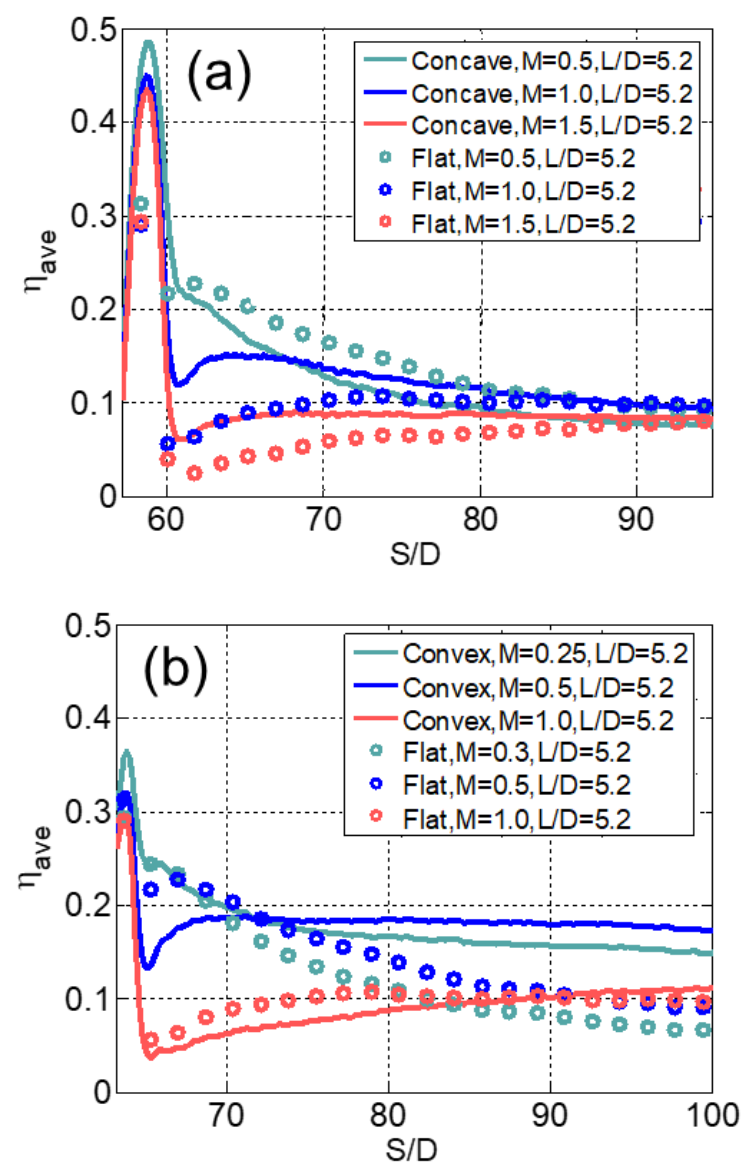

Fig. 18 Comparison of laterally averaged film cooling effectiveness of simple angle hole with $L / D=5.2$ with flat-plate data, (a) pressure side, (b) suction side.

Figure 19 shows the comparison of laterally averaged film cooling effectiveness of simple angle hole with $\mathrm{L} / \mathrm{D}=2.2$ with flat-plate data. This is used to show how the hole 
length-to-diameter ratio influences the curvature effect on the film cooling effectiveness. The LES results by the authors of simple angle hole with $\mathrm{L} / \mathrm{D}=2$ and 5 revealed that decreasing the hole length promotes the jet lift-off due to a more skewed hole exit velocity profile [33]. Fig. 19a shows that, on the pressure side, the film cooling effectiveness on the concave surface is slightly lower than the data on flatplate for all the blowing ratios, which is inconsistent with the variation trend of simple angle hole with $\mathrm{L} / \mathrm{D}=5$.2. This is associated with the variation of hole length, which changes the jet penetration features. At the low blowing ratio of $\mathrm{M}=0.5$, the jet still attaches the wall and the cross-stream pressure gradient pulls the jet away from the surface, decreasing the film cooling effectiveness. As the blowing ratio increases, the jet penetrates more deeply into the freestream and detaches the surface, which decreases the film cooling effectiveness. Since the jet lift-off is more severe for the $L / D=2.2$ than the $L / D=5.2$, the favorable influence of the centrifugal force, which pushes the jet into the wall, cannot offset the unfavorable influence of jet liftoff. Consequently, the film cooling effectiveness on the concave surface behaves quite identical to the flat-plate. In Fig. 19b, it is also observed that the favorable influence of the cross-stream pressure gradient, which pushes the jet into the wall at $\mathrm{M}=0.25$ and 0.5 , on the film cooling effectiveness is less pronounced than the hole with $\mathrm{L} / \mathrm{D}=5.2$. Still, at the blowing ratio of $\mathrm{M}=1.0$, the film cooling effectiveness on the convex surface behaves worse than the flat-plate.

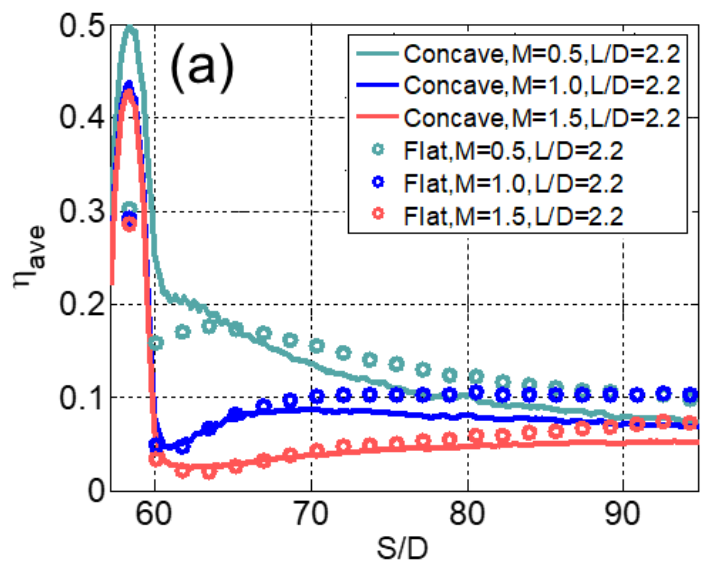

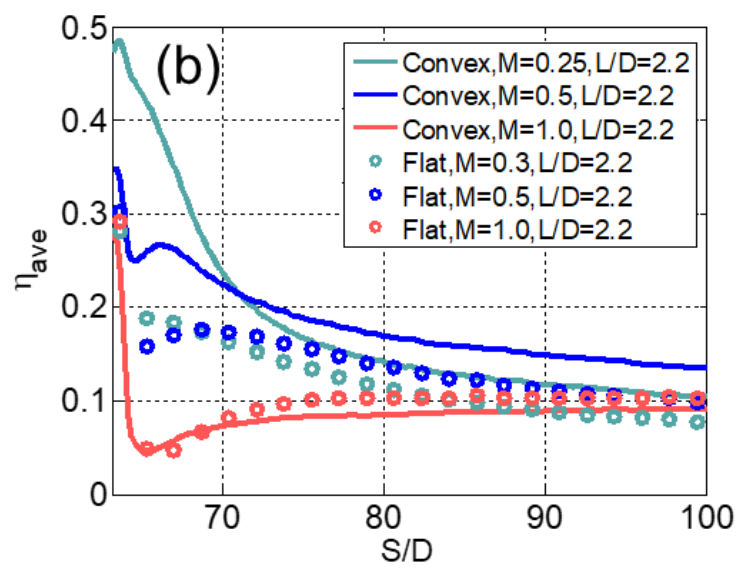

Fig. 19 Comparison of laterally averaged film cooling effectiveness of simple angle hole with $L / D=2.2$ with flat-plate data, (a) pressure side, (b) suction side.

The comparison of laterally averaged film cooling effectiveness of fan-shaped hole with $\mathrm{L} / \mathrm{D}=5.2$ with flatplate data is shown in Fig. 20. Figure 20a shows that the film cooling effectiveness on concave surface is slightly lower than the flat-plate data at $\mathrm{M}=0.5$ and 1.0 , while slightly higher than the flat-plate data at $\mathrm{M}=1.5$. The jet momentum at the hole exit is attenuated due to the hole expansion in lateral direction, which results in a reduced centrifugal force imposed on the jet in comparison with the cylindrical hole. This delays the point where the centrifugal force exceeds the force caused by the cross-stream pressure gradient for the fan-shaped hole. Consequently, the point lies between $\mathrm{M}=1.0$ and $\mathrm{M}=1.5$ for the fan-shaped hole, which is different from the point for the simple angle hole lying between $\mathrm{M}=0.5$ and $\mathrm{M}=1.0$. For the suction side, Fig. $20 \mathrm{~b}$ shows that the convex curvature favorably causes a marginal improvement on the film cooling effectiveness in comparison with the flat-plate data. This is because the jet is pushed into the wall since the centrifugal force is lower than the force caused by the cross-stream pressure gradient. Overall, the inclusion of shaped portion in the hole reduces the jet momentum and delays the point where the centrifugal force exceeds the force caused by the cross-stream pressure gradient. Also, the variation of film cooling effectiveness for the fan-shaped hole is less sensitive to surface curvature than the cylindrical holes. It can be concluded that the hole shape and hole $\mathrm{L} / \mathrm{D}$ can influence the curvature effect to different extent, since they influence the jet lift-off behaviors. 

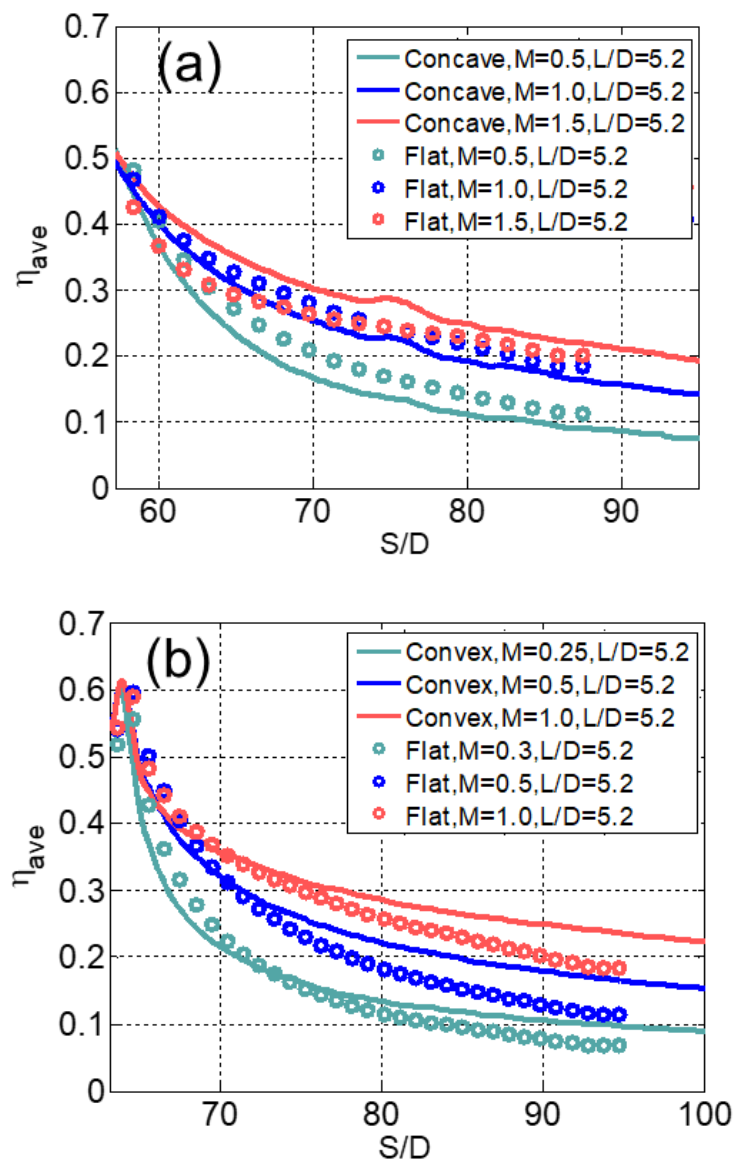

Fig. 20 Comparison of laterally averaged film cooling effectiveness of fan-shaped hole with $L / D=5.2$ with flat-plate data, (a) pressure side, (b) suction side.

\section{Conclusions}

The present study experimentally investigates the film cooling performance of a full-coverage film cooled turbine vane in a stationary and linear cascade. The tested blowing ratios for the showerhead, pressure side, and suction side range from 0.25 to 1.5 , with a density ratio of 1.5 . Geometrical parameters investigated included wall thickness (L/D from 2 to 5) and hole shape (simple angle hole, compound angle hole, and fan-shaped hole). The main conclusions are listed as follows:

(1) The showerhead cylindrical holes produce the highest film cooling performance at the blowing ratio of $\mathrm{M}=0.5$. Increasing the blowing ratio leads to jet lift-off and reduces the film cooling performance. The variation of showerhead film cooling effectiveness due to wall thickness reduction is generally marginal.

(2) For the suction side, as the blowing ratio increases, the film cooling effectiveness is reduced for the simple and compound angle holes, while increased for the fanshaped hole. Wall thickness variation has a limited influence on the film cooling effectiveness irrespective of hole shape. This is because the favorable influence caused by cross-stream pressure gradient and reduced near-wall turbulence compensates with the unfavorable influence due to wall thickness reduction.

(3) For the pressure side, the fan-shaped hole shows much higher film cooling effectiveness than the cylindrical holes, and the superiority becomes more pronounced as the blowing ratio increases. Increasing the wall thickness brings improvement by about 0.1-0.2 in film cooling effectiveness for simple angle hole and fanshape hole, while marginal improvement for the compound angle hole. Also the superposition method proposed by Sellers is validated. The geometrical variations, i.e., hole shape and wall thickness, exert limited influence on the application of the Sellers' method on the pressure side.

The surface curvature effect is discussed based on the film cooling effectiveness data of PS3 and SS3 rows of holes. Overall, the relationship between centrifugal force and the force caused by the cross-stream pressure gradient varies with wall thickness and hole shape. For the thick wall, the variation trend of simple angle hole with concave or convex curvature behaves differently with varying blowing ratios. The turning point for cylindrical hole lies between $\mathrm{M}=0.5$ and $M=1.0$. Decreasing the wall thickness narrows the gap between film cooling data on curved wall and flat wall for the simple angle hole due to more severe jet lift-off. The fanshaped hole dramatically reduces the sensitivity of film cooling effectiveness on wall curvature due to lower hole exit jet momentum, and consequently, the turning point lies between $\mathrm{M}=1.0$ and $\mathrm{M}=1.5$ for the fan-shaped hole. 


\section{Nomenclature}

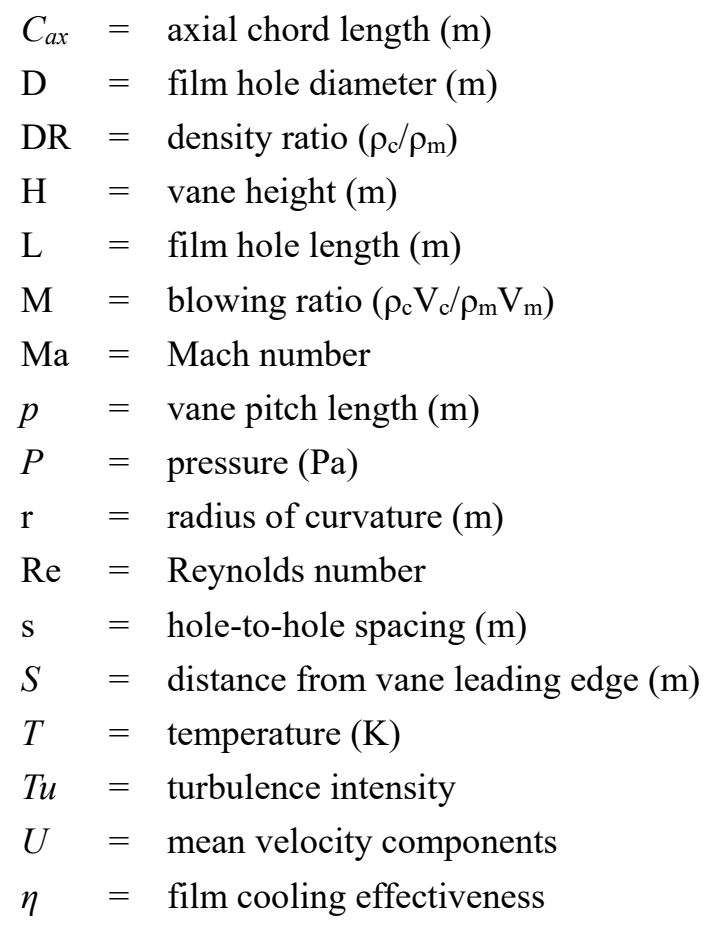

Subscripts

$$
\begin{aligned}
\text { ave } & =\text { lateral averaged } \\
c & =\text { coolant } \\
j & =\text { jet } \\
m & =\text { mainstream } \\
n & =\text { axis direction }
\end{aligned}
$$

\section{Reference}

[1] Liang, G., 2009, "Turbine airfoil with multiple near wall compartment cooling." U.S. Patent 7,556,476 B1.

[2] Li, W., Li, X., Ren, J., Jiang, H., Yang, L., and Ligrani, P., 2016, "Effect of Reynolds Number, Hole Patterns and Hole Inclination on Cooling Performance of an Impinging Jet Array: Part I-Convective Heat Transfer Results and Optimization," ASME J. Turbomach., 139(4), p. 041002.

[3] Li, W., Li, X., Ren, J., Jiang, H., and Yang, L., 2016, "Effect of Reynolds Number, Hole Patterns, Target Plate Thickness on Cooling Performance of an Impinging Jet Array: Part II-Conjugate Heat Transfer Results and Optimization," ASME J. Turbomach., 139(10), p.101001.

[4] Li, W., Xu, M., Ren, J. and Jiang, H., 2017, "Experimental Investigation of Local and Average Heat
Transfer Coefficients under an Inline Impinging Jet Array, Including Jets with Low Impingement Distance and Inclined Angle," ASME J Heat Transfer, 139(1), p.012201.

[5] Li, W., Lu, X., Li, X., Ren, J., and Jiang, H., 2018, "High resolution measurements of film cooling performance of simple and compound angle cylindrical holes with varying hole length-to-diameter ratio-Part I: Adiabatic film effectiveness," Int. J. Thermal Sci., 124, pp. 146-161.

[6] Lutum, E., and Johnson, B. V., 1999, "Influence of the Hole Length-to Diameter Ratio on Film Cooling With Cylindrical Holes," ASME J. Turbomach., 121, pp. 209-216.

[7] Harrington, M. K., McWaters, M. A., Bogard, D. G., Lemmon, C. A., and Thole, K. A., 2001, "FullCoverage Film Cooling With Short Normal Injection Holes," ASME Paper No. 2001-GT-0130.

[8] Drost, U., and Bolcs, A., 1998, "Investigation of Detailed Film Cooling Effectiveness and Heat Transfer Distributions on a Gas Turbine Airfoil," ASME Paper No. 98-GT-20.

[9] Ames, F. E., 1998, "Aspects of Vane Film Cooling With High Turbulence: Part II-Adiabatic Effectiveness," ASME J. Turbomach., 120, pp. 777 784.

[10] Cutbirth, J. M., and Bogard, D. G., 2002, "Evaluation of Pressure Side Film Cooling With Flow and Thermal Field Measurements, Part I: Showerhead Effects," ASME Paper No. GT-2002-30174.

[11] Cutbirth, J. M., and Bogard, D. G., 2002, "Evaluation of Pressure Side Film Cooling With Flow and Thermal Field Measurement, Part II: Turbulence Effects," ASME Paper No. GT-2002-30175.

[12] Sargison, J., Guo, S., Lock, G., Rawlinson, A., and Oldfield, M., 2002, "A Converging Slot-Hole FilmCooling Geometry_Part 2: Transonic Nozzle Guide Vane Heat Transfer and Loss," ASME J. Turbomach., 124(3), pp. 461-471.

[13] Guo, S., Lai, C., Jones, T., Oldfield, M., Lock, G., and Rawlinson, A., 1998, "The Application of Thin-Film Technology to Measure Turbine-Vane Heat Transfer and Effectiveness in a Film-Cooled, Engine-Simulated Environment," Int. J. Heat Fluid Flow, 19(6), pp. 594- 
600.

[14] Colban, W., Haendler, M., Gratton, A., and Thole, K., 2006, "Heat Transfer and Film-Cooling Measurements on a Stator Vane With Fan-Shaped Cooling Holes," ASME J. Turbomach., 128(1), pp. 53-61.

[15] Mhetras, S. P., Han, J. C., and Rudolph, R., 2007, "Effect of Flow Parameter Variation on Full Coverage Film Cooling Effectiveness for a Gas Turbine Blade," ASME Paper No. GT2007-27071.

[16] Zhang, L., Baltz, M., Pudupatty, R., and Fox, M., 1999, "Turbine Nozzle FilmCooling Study Using the Pressure Sensitive Paint (PSP) Technique," ASME Paper No. 99-GT-196.

[17] Zhang, L., and Pudupatty, R., 2000, "The Effects of Injection Angle and Hole Exit Shape on Turbine Nozzle Pressure Side Film-Cooling," ASME Paper No. 2000GT-0247.

[18] Nathan, M. L., Dyson, T. E., Bogard, D. G., and Bradshaw, S. D., 2014, "Adiabatic and Overall Effectiveness for the Showerhead Film Cooling of a Turbine Vane," ASME J. Turbomach., 136(3), p. 031005.

[19] Nadali, H. N., Karlsson, M., Utriainen, E., Kinell, M., and Wang, L, 2015, "Film-Cooling Performance of Multiple Arrays of Cylindrical and Fan-Shaped Holes", J. Propulsion and Power, 31(6), 1621-1630.

[20] Naik, S. , Krueckels, J. , Gritsch, M. , and Schnieder, M. , 2014, "Multirow Film Cooling Performances of a High Lift Blade and Vane," ASME J. Turbomach., 136(5), p. 051003.

[21] Gao, Z., Narzary, D. P., Mhetras, S., and Han, J. C., 2008, "Full-Coverage Film Cooling for a Turbine Blade With Axial-Shaped Holes," J. Thermophys. Heat Transfer, 22 (1), pp. 50-61.

[22] Han C, Ren J, Jiang H., 2014, "Experimental investigations of SYCEE film cooling performance on a plate and a tested vane of an F-class gas turbine". ASME Paper No. GT2014-25774.

[23] Russin, R. A., Alfred, D., and Wright, L. M., 2009, "Measurement of Detailed Heat Transfer Coefficient and Film Cooling Effectiveness Distributions Using PSP and TSP," ASME Paper No. GT2009-59975.

[24] Ahn, J., Mhetras, S., and Han, J. C., 2005, "FilmCooling Effectiveness on a Gas Turbine Blade Tip
Using Pressure-Sensitive Paint," ASME J. Heat Transfer, 127(5), pp. 521-530.

[25] Natsui, G. , Little, Z. , Kapat, J. S. , Dees, J. E. , and Laskowski, G. , 2015, “ A Detailed Uncertainty Analysis of Adiabatic Film Cooling Effectiveness Measurements Using Pressure Sensitive Paint," ASME Paper No. GT2015-42707.

[26] Li, X., Ren, J. and Jiang, H., 2016, "Influence of different film cooling arrangements on endwall cooling," Int. J. Heat Mass Transfer, 102, pp.348-359.

[27] Waye, S. K., and Bogard, D. G., 2006, "High Resolution Film Cooling Effectiveness Comparison of Axial and Compound Holes on the Suction Side of a Turbine Vane," ASME Paper No. GT2006-90225.

[28] Saumweber, C., Schulz, A., and Wittig, S., 2003, "FreeStream Turbulence Effects on Film Cooling With Shaped Holes," ASME J. Turbomach, 125, pp. 65-73.

[29] An, B. T., Liu, J. J., Zhou, S. J., 2017, “Geometrical Parameter Effects on Film-Cooling Effectiveness of Rectangular Diffusion Holes," ASME J. Turbomach., 139(8), 081010.

[30] Dittmar, J., Schulz, A. and Wittig, S., 2003, "Assessment of Various FilmCooling Configurations Including Shaped and Compound Angle Holes Based on Large-Scale Experiments", ASME J. Turbomach., 125 , pp. 57-64.

[31] Gao, Z., and Han, J. C., 2009, "Influence of Film-Hole Shape and Angle on Showerhead Film Cooling Using PSP Technique," ASME J. Heat Transfer, 131(6), p. 061701.

[32] Li, W., Lu, X., Li, X., Ren, J., and Jiang, H., 2018, "Geometrical Parameter Optimization Of A FanShaped Hole To Enhance Film Cooling Performance In Thin Wall Cooling," ASME Paper No. GT2018- 77232

[33] Li, W., W. Shi, X. Li, J. Ren, and H. Jiang ,2017, “On the Flow Structures and Adiabatic Film Effectiveness for Simple and Compound Angle Hole with Varied Length-to-Diameter Ratio by LES and PSP techniques", ASME J Heat Transfer, 139 (12), p.122201.

[34] Narzary, D. P., Liu, K. C., Rallabandi, A. P., and Han, J. C., 2012, "Influence of Coolant Density on Turbine Blade Film-Cooling Using Pressure Sensitive Paint Technique,” ASME J. Turbomach., 134(3), p. 031006.

[35] Y. Qin, P. Chen, J. Ren, Effects of wall curvature and 
streamwise pressure gradient on film cooling effectiveness, Appl. Therm. Eng. 107 (2016) 776-784

[36] Sellers, J. P., 1963, "Gaseous Film Cooling With Multiple Injection Stations,” AIAA J., 1(9), pp. 21542156.

[37] Ito, S., Goldstein, R. J., and Eckert, E. R. G., 1978, "Film Cooling of a Gas Turbine Blade," ASME J. Eng.
Power, 100, pp. 476-481.

[38] Schwarz, S. G., Goldstein, R. J., and Eckert, E. R. G., 1990, "The Influence of Curvature on Film Cooling Performance," ASME J. Turbomach., 100 , pp. 472478. 